\title{
Treatments of Advanced Non-Small Cell Lung Cancer (NSCLC) in an Italian Center: Drug Utilization and the Treatment Costs of Innovative Drugs
}

\author{
Francovito Piantedosi ${ }^{1}$, Raffaela Cerisoli ${ }^{1}$, Ciro Battiloro ${ }^{1}$, Francesca Andreozzi ${ }^{2}$, \\ Fabiana Vitiello ${ }^{1}$, Marina Gilli ${ }^{1}$, Valeria De Marino ${ }^{1}$, Antonietta Letizia ${ }^{1}$, \\ Antonella Bianco ${ }^{2}$, Ivana Caprice ${ }^{2}$, Adele Savoia ${ }^{3}$, Adriano Cristinziano $^{3}$, \\ Giorgia Smeraglio ${ }^{1}$, Danilo Rocco ${ }^{1}$ \\ UOC Pneumo-oncology, AORN Colli-Monaldi, Naples \\ Oncology, AORN Colli-Monaldi, Naples \\ Hospital Pharmacy, AORN Colli-Monaldi, Naples
}

\begin{abstract}
AIM: To provide an updated picture of the therapies most commonly used in the advanced Non-Small Cell Lung Cancer (NSCLC) setting, together with the relevant costs.

METHODS: This study considered the clinical records of patients affected by stage IIIb and IV NSCLC treated in the AORN dei Colli - Plesso Monaldi in Naples during the period January 2016-July 2017 and diagnosed since 2014, as well as the pathology lab database. Multivariate analyses were performed in order to identify the main predictors of time to next treatment and the main cost drivers.

RESULTS: Data were collected on 575 patients, who were mainly affected by adenocarcinoma (62\%) and squamous cell carcinoma (34\%). $64 \%$ of patients were reported having been tested for molecular biomarkers (among the patients tested, $13 \%$ were EGFR+, $4 \%$ Alk t, and 1\% ROS1 t). In accordance with the international guidelines, chemotherapy - as single agent or platinum-based doublets - was the prevalent first-line treatment, except among EGFR+ and ROS1 t patients, for whom the target therapy was authorized as first-line therapy. As second-line treatment, the target therapy and immune checkpoint inhibitors (nivolumab) were the most commonly used treatments. Drug expenditure per patient was remarkably higher in mutated patients $(€ 29,053)$ versus wild-type patients, or patients with unknown mutational status $(€ 11,854)$, who received just chemotherapy. The costs sustained in 2017 are proportionally higher than those sustained in 2016, mainly due to the increasing eligibility to target therapy and immune checkpoint inhibitors and the wider biomarker analysis performed. From multivariate analyses, among the predictors of a longer time to next treatment (TTNT) were a better performance status and target therapy both in first and second line. The therapy for squamous cell carcinoma and other nonadeno histotypes turned out to be less expensive in patients treated just in the first line than that for adenocarcinoma and adenosquamous carcinoma. The use of immune checkpoint inhibitors in the second line results in increased costs compared to the use of chemotherapy. Also the target therapy in the first line results in an increase in the total costs with respect to chemotherapy in patients who received just a first-line therapy.

CONCLUSIONS: Generally, in this study population, the treatments administered are in accordance with the international guidelines. The costs borne by the Health Systems are higher for the target therapy and the immune checkpoint inhibitors.
\end{abstract}

\section{Keywords}

Carcinoma; Non-Small Cell Lung Cancer; Economics; Mutation

\section{INTRODUCTION}

In Italy, lung cancer is among the most frequently diagnosed tumors, accounting for $11 \%$ of the new diagnoses of cancer [1], 85-90\% of which are attributable to smoking. The incidence is lower in females, especially in Southern Italy. 
The incidence is greater for Non-Small Cell Lung Cancers (NSCLCs) - mainly categorized as adenocarcinomas (39\% of all lung cancers), squamous cell carcinomas $(21 \%)$, and large-cell carcinomas (2\%) - followed by Small Cell Lung Cancers (SCLCs, 12\%).

In males, lung cancer is the first cause of cancer-related mortality $(26 \%)$, while in females it is the third cause of cancer-related mortality (11\%). This high mortality depends on a generally late diagnosis (which in most cases occurs at an advanced stage of disease), resulting in low life expectancy $(5$-year survival $=14.3 \%)$ and poor drug effectiveness.

While new screening methods are currently under study, a new arsenal of treatments (target therapies and immune checkpoint inhibitors) has been developed and recently introduced into the market, making it possible to achieve better outcomes, although curing advanced NSCLC is not yet possible.

In particular, over the last years, a small subset of patients, mainly affected by adenocarcinoma, benefited from therapies targeting mutations on Epidermal Growth Factor Receptor (EGFR), Anaplastic Lymphoma Kinase (ALK) translocation, and translocation in the receptor tyrosine kinase ROS proto-oncogene 1 (ROS1) [2].

Therapeutic algorithms are increasingly branched and the guidelines of the leading Scientific Societies need to be frequently updated [3-8].

In patients diagnosed with advanced NSCLC (stage IIIb-IV) and with ECOG Performance Status $=0-2$, chemotherapy with doublets of platinum-based third-generation drugs is recommended as first-line therapy (in patients without molecular drivers and with Programmed Death-Ligand 1 Tumor Proportion Score $<50 \%$ ). These patients, after 4 cycles and if the disease is well controlled, may receive a maintenance therapy with pemetrexed and bevacizumab. However, in the presence of EGFR mutations, which are detected in 10-15\% of Italian patients [8], the target therapy (TT) is carried out with tyrosine kinase inhibitors (TKIs), such as gefitinib, erlotinib, and afatinib as first-line therapy. In case of tumor progression, osimertinib may be administered in the presence of T790M mutation; otherwise, chemotherapy is used. In case of ALK translocation, crizotinib and alectinib are used in first line, and alectinib and ceritinib are used in second line. Crizotinib is also used in patients carrying a ROS1 translocation.

A new class of drugs - the immune checkpoint inhibitors - recently entered the market: they target the interaction between Programmed Death-Ligand 1 (PD-L1) in cancer cells and Programmed cell Death protein 1 PD-1 in T cells. When this interaction occurs, the response of the T cells against cancer cells is shut down: immune checkpoint inhibitors restore the $\mathrm{T}$ cells' ability to fight cancer cells. Initially developed in the melanoma setting, these agents are now used also in other types of hematological and solid cancers, including NSCLC [9]. Nivolumab, pembrolizumab, and atezolizumab have been available in Italy since March 2016, July 2017, and July 2018, respectively. Immune checkpoint inhibitors have become the standard of care for a subset of squamous and non-squamous NSCLC patients [2].

Pembrolizumab is the first-line treatment of choice in metastatic NSCLC patients whose cancer expresses PD-L1 with a Tumor Proportion Score (TPS) $\geq 50 \%$ and are negative for EGFR mutations, ALK, and ROS1 translocations [10], thus replacing chemotherapy in these patients. It is used as second-line therapy [10] if the tumor expresses PD-L1 with a Tumor Proportion Score (TPS) $\geq 1 \%$. In contrast, nivolumab and atezolizumab are indicated as second-line therapy, regardless of the PD-L1 expression [2].

As shown by the observational study LIFE [11], 40.4\% of NSCLC patients with advanced disease who finish the second-line therapy receive also a third-line treatment, which varies depending on the histology and mutation patterns, as well as the previous therapies received.

The AORN dei Colli - Plesso Monaldi in Naples is one of the reference centers for lung cancer in Italy.

This study considered the clinical records of patient affected by NSCLC at advanced stage (IIIb and IV) treated in the AORN dei Colli - Plesso Monaldi (Naples) over the time frame January 2016-July 2017, with the aim of providing a picture of the most commonly used therapies and the relevant costs.

During the study period, target therapies were already available, thereby resulting in a reliable real-world picture of the currently used therapy, keeping in mind that in this therapeutic area treatments change rapidly, and that during the study period some variations were made. For example, after AIFA's Resolution 252/2017 of February 2017 [12], the nivolumab indication was extended to cover not only squamous, but also non-squamous NSCLC.

Considering the recent therapeutic innovations in the treatment of NSCLC, multivariate analyses were performed in order to identify the main cost drivers. The economic impact of a more selective use of immune checkpoint inhibitors (after the detection of PD-L1 expression) is also discussed. 


\section{PATIENTS AND METHODS}

This study considered the clinical records of patient affected by NSCLC at advanced stage (the most frequently stage at the moment of diagnosis) treated in 3 Operative Units of the AORN dei Colli - Plesso Monaldi in Naples over the time frame January 2016-July 2017. Only patients diagnosed in and after 2014 were considered, because since 2014 molecular diagnosis is considered part of the routine diagnostic tests for NSCLC.

During this study, the VII edition of the TNM classification was used [13].

The data collected were managed through a unique and anonymous personal identification code, according to the rules of the Italian Medicines Agency [14]. Therefore, neither Ethics Committee's approval nor any informed consent were required. The study was notified to the local Ethics Committee of AORN Dei Colli on September $20^{\text {th }} 2018$, protocol number $18050 / 2018$

The data on tumor histology, grading, and mutational status were retrieved from the pathology lab database, while data on the pharmaceutical treatments and sequelae, as well as other demographic and essential clinical data (surgery, date of diagnosis, smoking status) were obtained from the patients' records.

Patients carrying PD-L1 mutation were generally categorized as wild-type (WT). Patients taking pembrolizumab $(n=1)$, approved just at the end of the study period, were excluded from the database. In case of inconsistencies, patients were eliminated from the database.

In order to determine the drug costs per patient, every patient was categorized as completed, ongoing, or lost, on the basis of their therapeutic status upon the closing of the database. The associated drug costs were calculated only in patients who had concluded the therapy, starting from the cost per mg or dosing unit, as defined by the hospital pharmacy.

Data on Progression Free Survival (PFS) were not available. Therefore, time to next treatment (TTNT) were taken as proxy of PFS. To be more precise, TTNT1 and TTNT2 are the time between diagnosis and therapy reassessment after failure of first or second line, respectively.

\section{STATISTICAL ANALYSES}

Multivariate linear regressions were performed, in order to evaluate the impact of baseline characteristics, patient status, and therapy on TTNT and total treatment costs.

For each dependent outcome, a complete model was at first considered, which included all patients characteristics: gender, age, performance status, duration of therapy, smoking status, staging, histotype, presence of mutations, status of the patients in therapy (ongoing first/ second/third line, having completed first/second/third line), and type of treatment for each line. Independent predictors were selected using LASSO (Least Absolute Shrinkage Selector Operator) regression [15] in order to define the best reduced model that predicts time to next treatment (TTNT1 and TTNT2) and total cost. Furthermore, multicollinearity was checked using the Variance Inflation Factor (VIF). If more than one parameter has VIF $>4$, the AIC criteria was used to choose the best model. To avoid confounding due to treatment interruption or patients lost to follow-up, the analysis on total cost was conducted, separately, on patients who didn't undergo second-line therapy patients (patients 1L), patients who didn't undergo third-line therapy (patients $2 \mathrm{~L}$ ), and patients who received a complete or partial third-line therapy (patients 3L). Reduced models are shown in this paper, while complete models are reported in the Supplementary files. All analyses were performed using the computer software R 3.1.2 [16].

\section{RESULTS}

\section{Pathology and Histology}

The database contained data about 577 patients diagnosed with advanced stage of NSCLC after 2014. For the purpose of this analysis, 1 adenocarcinoma patient was eliminated because treated with pembrolizumab and 1 patient was excluded due to a mistake in data (second-line treatment was a drug prescribed just in patients affected by small cell lung cancer).

Therefore, data analyzed were about 575 patients (Table I). 


\begin{tabular}{|c|c|c|c|}
\hline & & All patients $(n=575)$ & $\begin{array}{l}\text { New diagnoses January 2016-July } \\
\qquad 2017(n=439)\end{array}$ \\
\hline \multirow[t]{2}{*}{ Gender, n (\%) } & Female & $158(27 \%)$ & $118(27 \%)$ \\
\hline & Male & 417 (73\%) & $320(73 \%)$ \\
\hline \multirow{3}{*}{$\begin{array}{l}\text { Age at diagnosis, } \\
\text { mean (SD) }\end{array}$} & Female & $63.5(10.4)$ & $63.7(10.9)$ \\
\hline & Male & $65.8(8.6)$ & $65.8(8.4)$ \\
\hline & All & $65.2(9.2)$ & $65.2(9.2)$ \\
\hline \multirow{2}{*}{$\begin{array}{l}\text { Stage at } \\
\text { diagnosis, } \mathbf{n}(\%)\end{array}$} & IIIB & 165 (29\%) & $134(31 \%)$ \\
\hline & IV & $410(71 \%)$ & $304(69 \%)$ \\
\hline \multirow[t]{6}{*}{ Histology, n (\%) } & Adenocarcinoma & $356(62 \%)$ & $259(59 \%)$ \\
\hline & Squamous cell carcinoma & $194(34 \%)$ & $159(36 \%)$ \\
\hline & Lung carcinoma NOS & $20(3 \%)$ & $15(3 \%)$ \\
\hline & Adenosquamous carcinoma & $3(1 \%)$ & $3(1 \%)$ \\
\hline & Anaplastic carcinoma & $1(0 \%)$ & $1(0 \%)$ \\
\hline & Anaplastic sarcomatoid carcinoma & $1(0 \%)$ & $1(0 \%)$ \\
\hline \multirow[t]{2}{*}{ Surgery, n (\%) } & No & $424(74 \%)$ & $316(72 \%)$ \\
\hline & Yes & $151(26 \%)$ & $122(28 \%)$ \\
\hline \multirow{3}{*}{$\begin{array}{l}\text { Smoking habit, } \\
\text { n (\%) }\end{array}$} & Smoker & $224(39 \%)$ & $171(39 \%)$ \\
\hline & Former smoker & $283(50 \%)$ & $216(50 \%)$ \\
\hline & Nonsmoker & $64(11 \%)$ & $48(11 \%)$ \\
\hline \multirow[t]{5}{*}{ ECOG PS, n (\%) } & 0 & $236(41 \%)$ & $189(43 \%)$ \\
\hline & 1 & $260(45 \%)$ & $201(46 \%)$ \\
\hline & 2 & $60(10 \%)$ & $34(8 \%)$ \\
\hline & 3 & $11(2 \%)$ & $8(2 \%)$ \\
\hline & Missing & $8(1 \%)$ & $6(1 \%)$ \\
\hline \multicolumn{2}{|c|}{ Duration of follow-up, mean months (SD) } & $8.6(7.3)$ & $6.0(4.0)$ \\
\hline
\end{tabular}

Table I. Characteristics of patients with at least one visit during the time frame January 2016-July 2017

ECOG PS = Eastern Cooperative Oncology Group Performance Status; NOS = not otherwise specified; SD = standard deviation

\begin{tabular}{|c|c|c|c|c|}
\hline Histotype & Squamous & Adeno & Other & Total \\
\hline WT/UK, n (\%) & $29(7.9)$ & $265(71.8)$ & $10(2.7)$ & $304(82.4)$ \\
\hline EGFR mutation, $\mathrm{n}(\%)$ & $6(1.6)$ & $41(11.1)$ & $(0.0)$ & $47(12.7)$ \\
\hline ALK translocation, n (\%) & $0(0.0)$ & $16(4.3)$ & $(0.0)$ & $16(4.3)$ \\
\hline ROS1 translocation, n (\%) & $0(0.0)$ & $2(0.5)$ & $(0.0)$ & $2(0.5)$ \\
\hline Total, n (\%) & $35(9.5)$ & $324(87.8)$ & $10(2.7)$ & $369(100.0)$ \\
\hline
\end{tabular}

Table II. Biomolecular determination in the whole database $(n=575)$. Only patients who had undergone histotype determination $(n=369)$ were included. "Adeno" groups both adenocarcinoma and adenosquamous carcinoma histotypes

ALK = Anaplastic Lymphoma Kinase; EGFR = Epidermal Growth Factor Receptor; WT/UK = wild-type and unknown

The study considered two different populations:

- all the patients recorded in the database $(n=575)$;

- incident cases during the observation period $(n=439)$.

The data collected show that $73 \%$ of patients were male and that the mean age was 65 . In 2017, histology for NSCLC cases was defined mainly via bioptic material and cytological samples. The main histotypes were:

- adenocarcinoma: $62 \%$;

- squamous cell carcinoma: $34 \%$.

Other histotypes were not otherwise specified (NOS), adenosquamous carcinoma, anaplastic carcinoma, and anaplastic sarcomatoid carcinoma.

Considering the whole database, $26 \%$ of patients underwent surgery, $89 \%$ were current or former smokers. The mean duration of follow-up was 9 months.

Considering all patients in the database, $64 \%$ of patients were reported having been tested for EGFR, ALK mutation and, more recently (since 2017), ROS1 and PD-L1. Among the samples analyzed, 13\% had EGFR mutation, 4\% had ALK, and 1\% had ROS1. According to 
the pathology lab data, the prevalence of EGFR, ALK, and ROS1 mutations equals to $18 \%$ of the series analyzed (Table II).

Molecular characterization was performed in a low percentage of squamous histotypes (18\%). On the contrary, the test rate for adeno histotypes (always including adenocarcinoma and adenosquamous carcinoma in this article) was $88 \%$ (Table II).

Among the patients diagnosed in the period 2016-2017 $(n=439)$ : EGFR mutation was found in $11 \%$, ALK in $4 \%$, and ROS1 in $1 \%$. Preliminary data on a subset of patients tested for PD-L1 $(n=33)$ showed a level above $1 \%$ in $63 \%$ of them.

\section{Therapeutic Regimens}

Figure 1 and Table III show the therapeutic regimens used in the study period in patients diagnosed since 2014 in the main histotypes only: adeno and squamous cell carcinoma.

Table I-suppl, in the Supplementary files, provides a complete panorama about therapeutic regimens in non-surgical patients.

In patients with advanced stages of disease (IIIb-IV), chemotherapy - as single agent or platinum-based doublets - is the prevalent first-line treatment, except among EGFR ${ }^{+}$and ROS1 $\mathrm{t}$ patients, for whom TKIs were authorized as first-line therapy. On the contrary, as for second-line treatment, target therapy and immune checkpoint inhibitors (nivolumab) were the most commonly used.

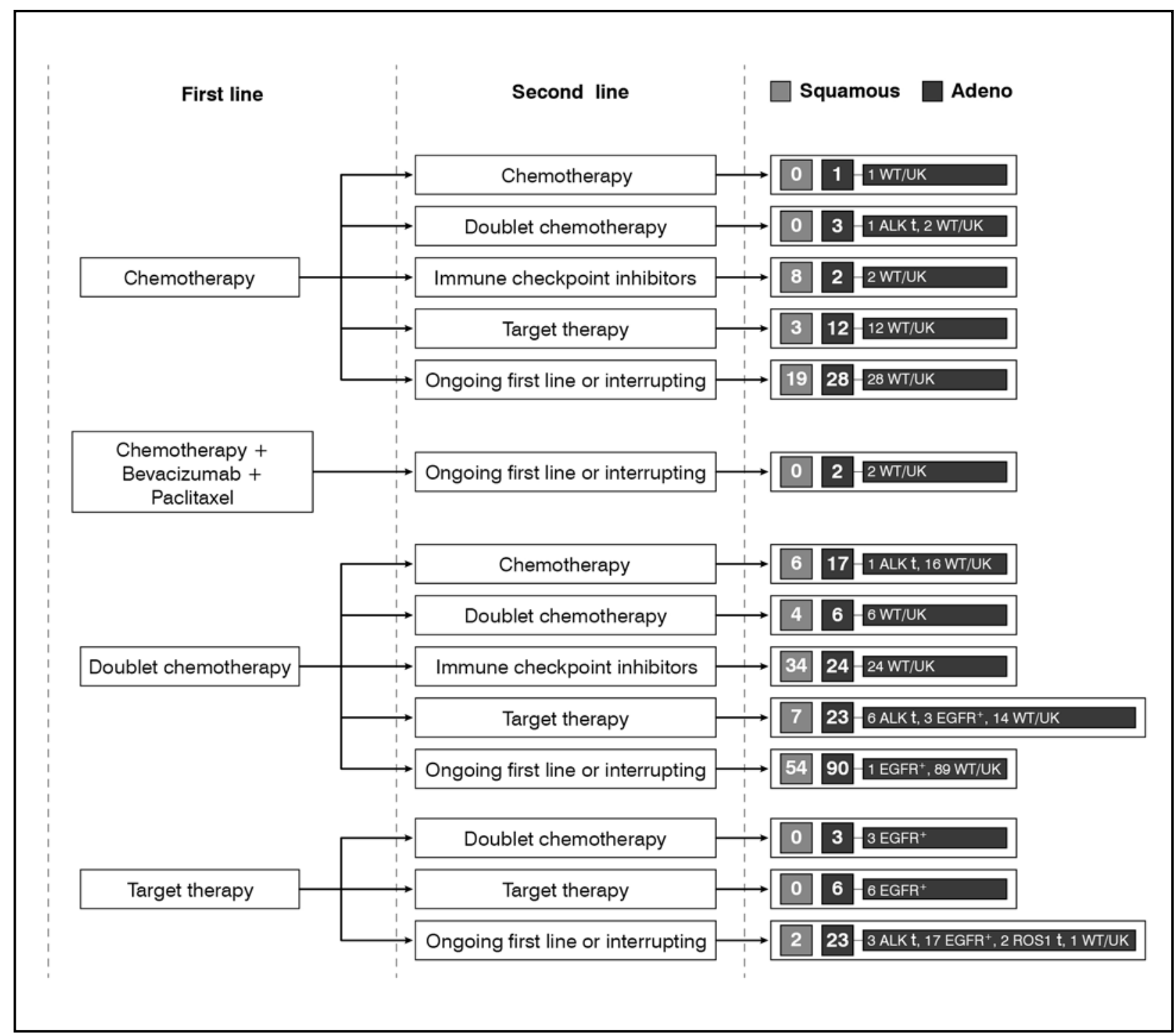

Figure 1. Therapeutic regimens in non-surgical patients affected by adenocarcinoma and adenosquamous carcinoma (together labelled as "adeno") and squamous cell carcinoma. In addition, 6 patients (1 squamous and 5 adeno) took Best Supportive Care (data not shown)

$\mathrm{ALK} \mathrm{t}=$ Anaplastic Lymphoma Kinase translocation; $\mathrm{EGFR}^{+}=$Epidermal Growth Factor Receptor mutation; interrupting = main reasons for interrupting drug treatment were: worsened health conditions, no information available, gone to radiotherapy, disease progression; ROS1 $t=$ ROS1 translocation; WT/ UK = wild-type and unknown 


\begin{tabular}{|c|c|c|c|}
\hline & Adeno & Squamous & Total \\
\hline CT, n (\%) & $41(33)$ & $13(19)$ & $54(28)$ \\
\hline Single agent CT, $n(\%)$ & $27(66)$ & $9(69)$ & $36(67)$ \\
\hline - Docetaxel & $19(70)$ & $6(67)$ & $25(69)$ \\
\hline - Gemcitabine & $6(22)$ & $0(0)$ & $6(17)$ \\
\hline - Pemetrexed & $0(0)$ & $1(11)$ & $1(3)$ \\
\hline - Vinorelbine & $1(4)$ & $2(22)$ & $3(8)$ \\
\hline - Vinorelbine os & $1(4)$ & $0(0)$ & $1(3)$ \\
\hline CTD, n (\%) & $14(34)$ & $4(31)$ & $18(33)$ \\
\hline - Carboplatin-gemcitabine & $3(21)$ & $1(25)$ & $4(22)$ \\
\hline - Carboplatin-pemetrexed & $4(29)$ & $2(50)$ & $6(33)$ \\
\hline - Carboplatin-vinorelbine & $0(0)$ & $1(25)$ & $1(6)$ \\
\hline - Cisplatin-docetaxel & $1(7)$ & $0(0)$ & $1(6)$ \\
\hline - Cisplatin-gemcitabine & $1(7)$ & $0(0)$ & $1(6)$ \\
\hline - Cisplatin-pemetrexed & $5(36)$ & $0(0)$ & $5(28)$ \\
\hline ICI, n (\%) & $34(27)$ & $47(67)$ & $81(42)$ \\
\hline - Nivolumab & $34(100)$ & $47(100)$ & $81(100)$ \\
\hline TT, n (\%) & $50(40)$ & $10(14)$ & $60(31)$ \\
\hline - Afatinib & $1(2)$ & $1(10)$ & $2(3)$ \\
\hline - Crizotinib & $10(20)$ & $0(0)$ & $10(17)$ \\
\hline - Erlotinib & $31(62)$ & $8(80)$ & $39(65)$ \\
\hline - Gefitinib & $2(4)$ & $1(10)$ & $3(5)$ \\
\hline - Osimertinib & $6(12)$ & $0(0)$ & $6(10)$ \\
\hline Overall total, n (\%) & $125(100)$ & $70(100)$ & $195(100)$ \\
\hline
\end{tabular}

Table III. Completed and ongoing second-line treatments (only adeno - including adenocarcinoma and adenosquamous carcinoma and squamous histotypes, $n=195$ ), also including operated patients

$\mathrm{CT}=$ chemotherapy; $\mathrm{CTD}=$ doublet chemotherapy; $\mathrm{ICI}=$ immune checkpoint inhibitors; $\mathrm{TT}=$ target therapy

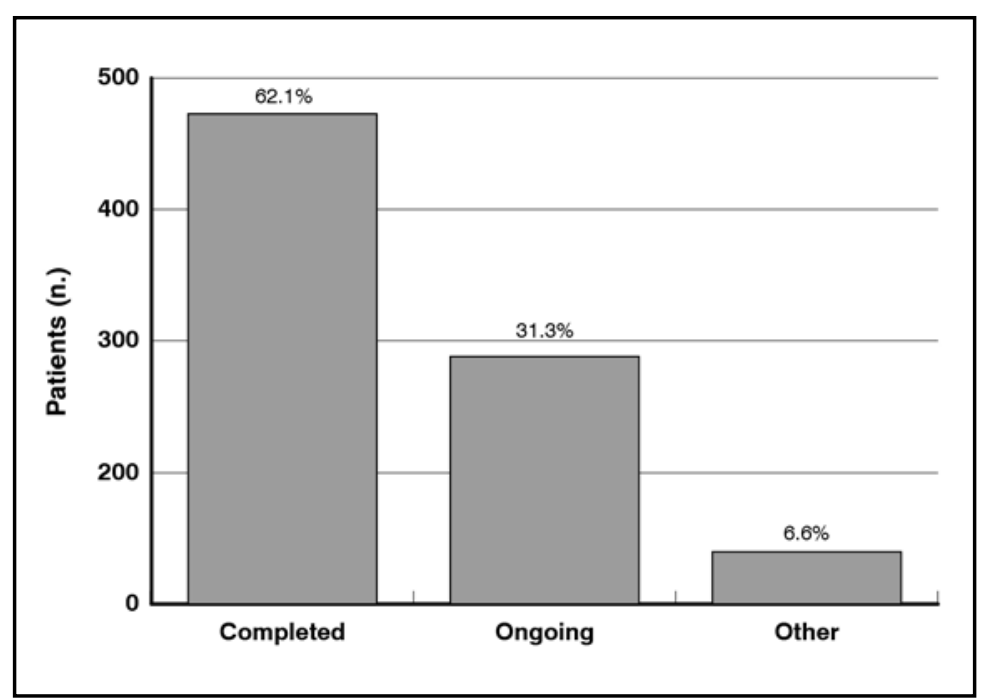

Figure 2. Number of patients per status upon closing of the database (July 2017)
Table III confirms that immune checkpoint inhibitors were used more than chemotherapy as second-line therapy, especially in squamous cell carcinoma.

Upon the database lock (July 2017), patients with at least one follow-up who had completed one line of treatment were $62.1 \%, 31.3 \%$ were still on treatment, while $6.6 \%$ of patients were waiting for supportive care, or a chemotherapy line, or radiotherapy (Figure 2).

The reasons for the discontinuation of treatment are reported in Tables IV (first-line therapy) and V (second-line therapy).

Both in the first- and second-line treatment, progression of disease and worsened health conditions were the main reasons for treatment discontinuation, the first accounting for more than a half of the causes of discontinuation. Despite the significant AEs generally associated with chemotherapy, the backbone of first-line treatment, toxicity is reported to be the reason for discontinuation only in $6.5 \%$ of the patients in first-line treatment.

\section{Pharmaceutical Expenditure}

The pharmaceutical expenditure associated with drugs was calculated, as reported in Table VI.

The total cost for treatments was $€ 6.5$ million. In considering the expenditure per patient, some important differences appear. Patients who have completed the first-line therapy (chemotherapy) are associated with a lower pharmaceutical expenditure, while patients in 
second- and third-line are associated with a 2- or 3-fold increase in the expenditure.

In addition, ongoing patients result in a greater expenditure per patient, compared with completed patients.

Also after adjustment for therapy duration, the cost per patient-month in patients who completed the first line of therapy is lower than the cost for other groups, and the cost per patient-month for ongoing patients is higher than the cost for completed patients.

Expenditure per patient is remarkably higher in mutated patients, versus wild-type patients or patients with unknown mutational status, who receive chemotherapy only (Table VII).

This is mainly due to longer therapy since, after adjustment for length of treatment, the cost per patient-month results similar between mutated and wild-type/unknown patients, except for patients carrying ALK mutation, whose cost remains higher. In the current scenario, patients eligible for target therapy and immune checkpoint inhibitors are increasing, thus also the pharmaceutical expenditure increases (Figure 3).

Nivolumab - the only immune checkpoint inhibitor authorized as second line upon the closing of the database - accounts for $66 \%$ of the expenditure for second-line treatments $(€ 1,689,179)$, with a mean cost per patient treated of almost $€ 20,000$ (several treatments were ongoing, therefore the mean cost is provisional). The expenditure for TKIs accounts for $30 \%$ of the expenditure for second-line treatments, with $€ 775,638$ spent for 62 patients (about $€$ 12,500 per patient).

In third line, 19 patients were treated with an immune checkpoint inhibitor (nivolumab), accounting for $88 \%$ of the expenditure for third-line therapy ( $€$ 363,735

\begin{tabular}{lc}
\hline \multicolumn{1}{c}{ Reasons for first-line treatment interruption } & $\mathbf{N}(\%)$ \\
\hline NO & $\mathbf{1 8 4} \mathbf{( 3 3 . 2 7 )}$ \\
YES & $\mathbf{3 6 9}(\mathbf{6 6 . 7 3 )}$ \\
- PD & $223(60.43)$ \\
- Worsened health conditions & $76(20.60)$ \\
- Toxicity & $24(6.50)$ \\
- Death & $16(4.34)$ \\
- Stability & $7(1.90)$ \\
- Lost at follow-up & $7(1.90)$ \\
- Surgery & $3(0.81)$ \\
- Remission & $3(0.81)$ \\
- NA & $3(0.81)$ \\
- Moved abroad & $2(0.54)$ \\
- Therapy switch & $1(0.27)$ \\
- Gone to RT & $1(0.27)$ \\
- Partial response & $1(0.27)$ \\
- End of cycle & $1(0.27)$ \\
- Adj CT & $1(0.27)$ \\
\hline
\end{tabular}

Table IV. Reasons for first-line treatment discontinuation

Adj $C T=$ adjuvant chemotherapy; $\mathrm{NA}=$ not assessed; $\mathrm{PD}=$ progression of disease $\mathrm{RT}=$ radiotherapy

\begin{tabular}{lc}
\hline \multicolumn{1}{l}{ Reasons for second-line treatment interruption } & $\mathbf{N}(\%)$ \\
\hline NO & $\mathbf{1 0 8}(\mathbf{5 2 . 6 8 )}$ \\
YES & $\mathbf{9 7}(\mathbf{4 7 . 3 2 )}$ \\
- PD & $56(57.73)$ \\
- Worsened health conditions & $21(21.65)$ \\
- Death & $9(9.28)$ \\
- Toxicity & $4(4.12)$ \\
- End of cycle & $3(3.09)$ \\
- Lost at follow-up & $2(2.06)$ \\
- Gone to RT & $1(1.03)$ \\
- Starting TKI & $1(1.03)$ \\
Overall total & $\mathbf{2 0 5 ( 1 0 0 . 0 0 )}$ \\
\hline
\end{tabular}

Table V. Reasons for second-line treatment discontinuation.

$\mathrm{PD}$ = progression of disease; $\mathrm{RT}$ = radiotherapy; TKI = Tyrosine Kinase Inhibitors

\begin{tabular}{|c|c|c|c|c|c|}
\hline Status & $\mathbf{N}$ & $\begin{array}{c}\text { Length of } \\
\text { therapy (months) }\end{array}$ & $\begin{array}{c}\text { Total expenditure } \\
(\boldsymbol{(})\end{array}$ & $\begin{array}{l}\text { Expenditure per } \\
\text { patient (€) }\end{array}$ & $\begin{array}{c}\text { Expenditure per } \\
\text { patient-month }(€)\end{array}$ \\
\hline Completed & 361 & 8.4 & $3,190,954$ & 9,065 & 1,077 \\
\hline $1^{\text {st line }}$ & 200 & 5.3 & 746,668 & $3,909^{1}$ & 736 \\
\hline $1^{\text {st line/maintenance therapy }}$ & 32 & 9.8 & 375,433 & 11,732 & 1,199 \\
\hline $2^{\text {nd }}$ line & 103 & 11.8 & $1,412,420$ & 13,713 & 1,158 \\
\hline $3^{\text {rd }}$ line & 26 & 17.0 & 656,434 & 25,247 & 1,482 \\
\hline Ongoing & 173 & 9.7 & $3,316,753$ & 19,396 & 1,996 \\
\hline $1^{\text {st line }}$ & 81 & 4.6 & 458,130 & $5,799^{2}$ & 1,265 \\
\hline $1^{\text {st }}$ line/maintenance therapy & 16 & 10.0 & 323,236 & 20,202 & 2,017 \\
\hline $2^{\text {nd }}$ line & 62 & 14.4 & $1,872,491$ & 30,201 & 2,099 \\
\hline $3^{\text {rd }}$ line & 14 & 18.3 & 662,895 & 47,350 & 2,586 \\
\hline Overall total & 534 & 8.8 & $6,507,707$ & 12,443 & 1,409 \\
\hline
\end{tabular}

Table VI. Pharmaceutical expenditure per status

${ }^{1}$ Total expenditure not reported in 9 patients

${ }^{2}$ Total expenditure not reported in 2 patients 


\begin{tabular}{lccccc}
\hline \multicolumn{1}{c}{ Mutational status } & $\mathbf{N}$ & $\begin{array}{c}\text { Length of } \\
\text { therapy (months) }\end{array}$ & $\begin{array}{c}\text { Total expenditure } \\
(\boldsymbol{\epsilon})\end{array}$ & $\begin{array}{c}\text { Expenditure per } \\
\text { patient }(\boldsymbol{\epsilon})\end{array}$ & $\begin{array}{c}\text { Expenditure per } \\
\text { patient-month }(\boldsymbol{\epsilon})\end{array}$ \\
\hline Mutated (ALK and EGFR) & $\mathbf{4 6}$ & $\mathbf{1 5 . 0}$ & $\mathbf{1 , 3 3 6 , 4 2 1}$ & $\mathbf{2 9 , 0 5 3}$ & $\mathbf{1 , 9 4 2}$ \\
ALK & 14 & 12.3 & 572,291 & 40,878 & 3,336 \\
EGFR & 32 & 16.1 & 764,131 & 23,879 & 1,479 \\
UK and WT & $\mathbf{3 9 7}$ & $\mathbf{9 . 0}$ & $\mathbf{4 , 5 9 9 , 4 1 8}$ & $\mathbf{1 1 , 8 5 4}$ & $\mathbf{1 , 3 1 6}$ \\
WT/UK & 167 & 9.0 & $1,761,141$ & 10,805 & 1,201 \\
WT & 230 & 9.0 & $2,838,278$ & $12,615^{2}$ & 1,399 \\
Overall total & $\mathbf{4 4 3}$ & $\mathbf{9 . 6}$ & $\mathbf{5 , 9 3 5 , 8 4 0}$ & $\mathbf{1 3 , 6 7 7}$ & $\mathbf{1 , 4 2 0}$ \\
\hline
\end{tabular}

Table VII. Pharmaceutical expenditure per mutational status for patients who completed at least one line of therapy

${ }^{1}$ Total expenditure not reported in 4 patients

${ }^{2}$ Total expenditure not reported in 5 patients

ALK = Anaplastic Lymphoma Kinase; EGFR = Epidermal Growth Factor Receptor; UK = unknown; WT = wild-type

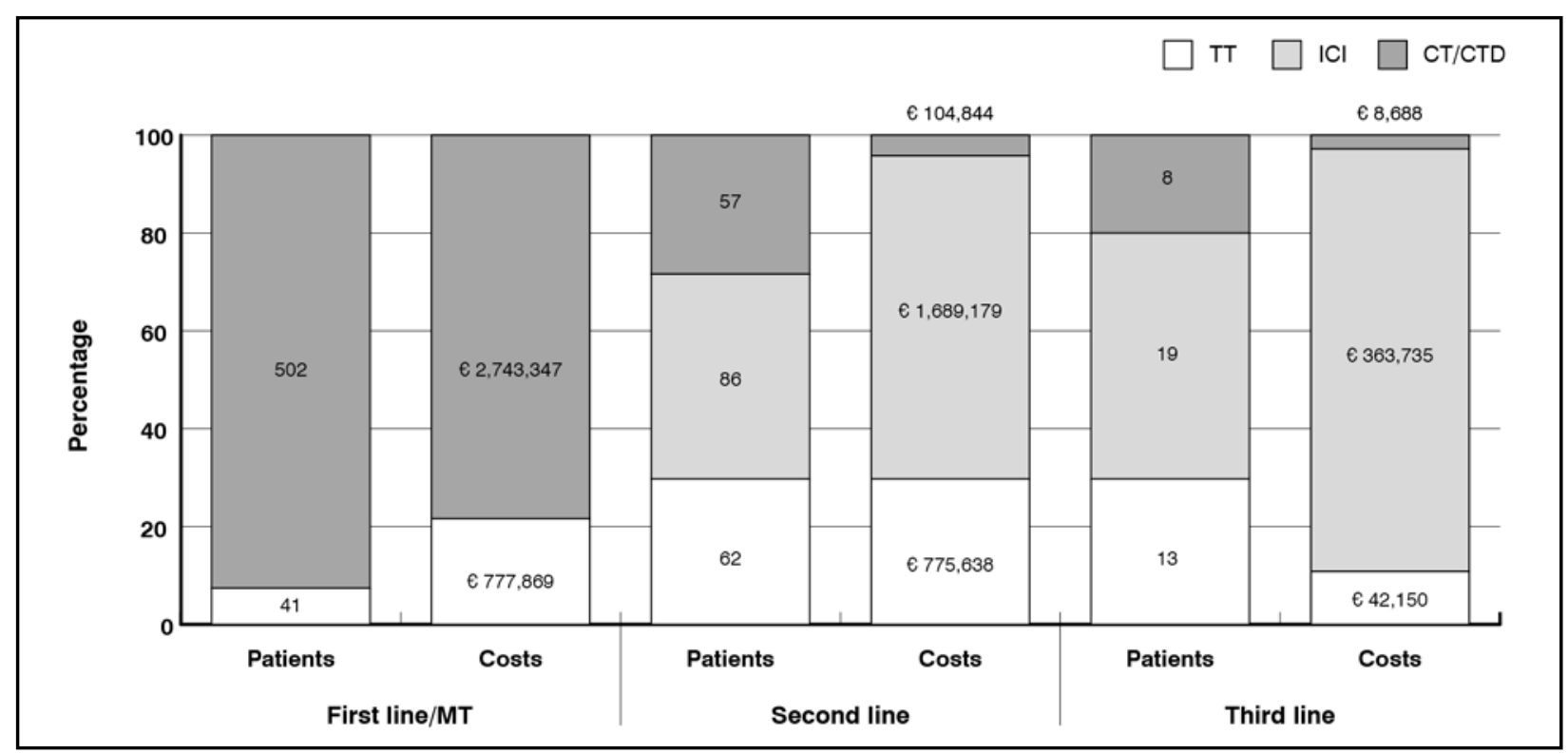

Figure 3. Total expenditure considering each separate line of therapy. Per each line of therapy, the left column represents the number of patients treated with each type of therapy, while the right column reports the relative expenditure.

$\mathrm{CT}=$ chemotherapy; CTD = doublet chemotherapy; $\mathrm{ICI}=$ immune checkpoint inhibitors; $\mathrm{MT}=$ maintenance therapy; $\mathrm{TT}=$ target therapy

\begin{tabular}{|c|c|c|c|c|}
\hline \multirow{2}{*}{ TTNT1 $(n=190)$} & \multicolumn{4}{|c|}{ Reduced model } \\
\hline & \multicolumn{3}{|c|}{ Effect $(95 \% \mathrm{Cl})$} & p-value \\
\hline Male & -1.25 & -3.04 & 0.54 & 0.17 \\
\hline Maintenance & 4.83 & 2.86 & 6.80 & $<0.001$ \\
\hline TT vs chemo $1 \mathrm{~L}$ & 8.06 & 4.27 & 11.85 & $<0.001$ \\
\hline
\end{tabular}

Table VIII. Results of the multivariate analysis of the TTNT1. Only a reduced model, after a LASSO regression, is reported. All variables with a $p$ value $<0.05$ are statistically associated with TTNT1

$1 \mathrm{~L}=$ patients who didn't undergo second-line therapy; TT = target therapy; TTNT1 = time between diagnosis and therapy reassessment after failure of first-line treatment out of $€ 414,572$ ), while TKIs were used in 13 patients, for a total of $€ 42,150$ (several treatments were still ongoing upon the closing of the database).

Even though only the first 7 months of 2017 are considered, the expenditure for immune checkpoint inhibitors has greatly increased, in comparison with the previous year ( $€ 229,061$ in 2016 vs $1,823,853$ in the period January-July 2017). The expenditure for chemotherapy and target therapy in 2016 was $€ 1,329,524$ and $€ 864,467$, respectively, while in the first seven months of 2017 was $€ 1,234,507$ and $€ 596,887$, respectively.

\section{MULTIVARIATE ANALYSES}

According to the multivariate analyses, receiving maintenance therapy results in a 4.83-month longer TTNT1 (Table VIII; see Table II-suppl, in the Supplementary files to view the complete and the reduced model together). Furthermore, starting TT in first line is associated with a longer duration of treatment with respect to chemotherapy ( +8.06 months).

TTNT2 was higher in better ECOG PS (Table IX; see Table III-suppl, in the Supplementary files to view the complete and the reduced model together). Finally, being eligible for TKIs both in first line and after the first progression of disease increases TTNT. 
The multivariate analysis of total costs (Table X; see Table IV-suppl, in the Supplementary files to view the complete and the reduced model together) shows that every additional month of therapy is associated with a cost increase ranging from $€ 661$ in first line up to $€ 1,383$ for patients who received a complete or partial third-line therapy (3L).

A trend toward a cost increase from patients $1 \mathrm{~L}$ to patients $3 \mathrm{~L}$ is shown also in those ongoing treatment with respect to those that completed the therapy. The therapy for squamous cell carcinoma and other non-adeno histotypes costs less than that for adenocarcinoma and adenosquamous carcinoma for patients in first line. The same trend is observed for patients who didn't undergo thirdline therapy $(2 \mathrm{~L})$ and $3 \mathrm{~L}$, but the savings are not statistically significant. In patients $2 \mathrm{~L}$ and $3 \mathrm{~L}$, cost of mutated patients is higher than that of WT or unknown.

The use of target therapy in first line results in increased costs with respect to chemotherapy, while for patients $2 \mathrm{~L}$ and $3 \mathrm{~L}$ there is no cost difference between target therapy and chemotherapy used in second line. The use of immune checkpoint inhibitors in second line increases costs both for patients $2 \mathrm{~L}$ and $3 \mathrm{~L}$, compared to the use of chemotherapy. Third line treatments do not affect the total treatment cost; in fact total costs according to specific class

\begin{tabular}{|c|c|c|c|c|c|c|c|c|c|c|c|c|}
\hline \multirow[b]{3}{*}{ Male } & \multicolumn{4}{|c|}{ Patients 1L $(n=312)$} & \multicolumn{4}{|c|}{ Patients 2L $(n=154)$} & \multicolumn{4}{|c|}{ Patients 3L $(n=40)$} \\
\hline & \multirow[t]{2}{*}{ Mean } & \multicolumn{2}{|c|}{$95 \% \mathrm{Cl}$} & \multirow[t]{2}{*}{$\mathbf{p}$} & \multirow[t]{2}{*}{ Mean } & \multicolumn{2}{|c|}{$95 \% \mathrm{Cl}$} & \multirow[t]{2}{*}{$\mathbf{p}$} & \multirow{2}{*}{$\begin{array}{c}\text { Mean } \\
-8,751\end{array}$} & \multicolumn{2}{|c|}{$95 \% \mathrm{Cl}$} & \multirow[t]{2}{*}{$\mathbf{p}$} \\
\hline & & & & & & & & & & $-22,398$ & 4,896 & \\
\hline Age (per year) & -34 & -91 & 23 & & & & & & & & & \\
\hline PS 1 vs 0 & 584 & -547 & 1,714 & & & & & & 9,649 & $-3,347$ & 22,645 & \\
\hline PS 2 vs 0 & -396 & $-2,342$ & 1,550 & & & & & & 4,888 & $-21,222$ & 30,998 & \\
\hline PS 3 vs 0 & 907 & $-3,195$ & 5,009 & & & & & & & & & \\
\hline $\begin{array}{l}\text { Length of therapy } \\
\text { (months) }\end{array}$ & 661 & 541 & 780 & $* * *$ & 1,050 & 711 & 1,388 & $* \star *$ & 1,383 & 628 & 2,137 & *** \\
\hline $\begin{array}{l}\text { Former smoker vs } \\
\text { nonsmoker }\end{array}$ & 1,569 & -159 & 3,297 & $\circ$ & $-4,116$ & $-13,258$ & 5,026 & & 6,211 & $-11,708$ & 24,130 & \\
\hline Smoker vs nonsmoker & 1,473 & -271 & 3,217 & $\circ$ & $-4,744$ & $-14,537$ & 5,049 & & 7,842 & $-13,688$ & 29,373 & \\
\hline Stage III vs IV & & & & & 8,988 & 3,280 & 14,696 & ** & $-10,347$ & $-24,161$ & 3,466 & \\
\hline $\begin{array}{l}\text { Other histotype vs } \\
\text { adeno }\end{array}$ & $-5,519$ & $-8,459$ & $-2,579$ & $* * *$ & $-8,451$ & $-20,246$ & 3,344 & & $-12,232$ & $-29,127$ & 4,664 & \\
\hline Squamous vs adeno & $-4,015$ & $-5,174$ & $-2,856$ & $* * *$ & $-2,442$ & $-8,794$ & 3,910 & & & & & \\
\hline Mutated vs WT/UK & & & & & 10,069 & 923 & 19,214 & * & 17,256 & 870 & 33,641 & * \\
\hline $\begin{array}{l}\text { Ongoing vs completed } \\
\text { therapy }\end{array}$ & 3,062 & 1,882 & 4,242 & $* * *$ & 9,316 & 2,691 & 15,941 & ** & 28,904 & 14,734 & 43,074 & *** \\
\hline Maintenance & 6,231 & 4,637 & 7,824 & $* * *$ & 4,352 & $-4,121$ & 12,826 & & 5,767 & $-12,560$ & 24,094 & \\
\hline TT vs chemo $1 \mathrm{~L}$ & 5,977 & 4,037 & 7,916 & $* * *$ & & & & & & & & \\
\hline $\mathrm{ICl}$ vs chemo $2 \mathrm{~L}$ & & & & & 11,300 & 3,583 & 19,016 & $* *$ & 25,202 & 8,106 & 42,297 & ** \\
\hline TT vs chemo 2L & & & & & 3,673 & $-3,872$ & 11,217 & & 1,447 & $-14,464$ & 17,359 & \\
\hline Adjusted $\mathrm{R}^{2}$ & & 0.6458 & & $\star * *$ & & 0.428 & & $\star \star \star *$ & & 0.6667 & & *** \\
\hline
\end{tabular}

Table X. Results of the multivariate analysis of total costs. Only a reduced model, after a LASSO regression, is reported. Patients who received radiotherapy were not considered. All variables with a $p$ value $<0.05$ are statistically associated with total costs. "Adeno" includes both adenocarcinoma and adenosquamous carcinoma

*** $p$-value $<0.001,{ }^{* *} p$-value $<0.01,{ }^{*} p$-value $<0.05,{ }^{\circ} p$-value $<0.1$

$1 \mathrm{~L}=$ patients who didn't undergo second-line therapy; $2 \mathrm{~L}=$ patients who didn't undergo third-line therapy; $3 \mathrm{~L}=$ patients who received a complete or partial third-line therapy; ICl = immune checkpoint inhibitors; PS = perfomance status; TT = target therapy; UK = unknown; WT = wild-type; 
therapy (chemotherapy, target therapy and immune checkpoint inhibitors) are similar (data not shown).

For patients $3 \mathrm{~L}$, third-line treatment did not result a predictor of costs.

\section{DISCUSSION}

This study was carried on in order to provide updated information about the epidemiology, therapy used, and costs of advanced NSCLC in Italy since the introduction of immune checkpoint inhibitors. Since treatments are rapidly changing - and more in this field than in others - and the guidelines need to be updated very frequently, recent data must be used in order to obtain a reliable picture of the drugs used and the real costs incurred today by the healthcare systems.

In this study, adenocarcinoma was detected in $62 \%$ of patients, while $34 \%$ had squamous cell carcinoma.

LIFE [11] - an Italian study taking into account NSCLC patients with advanced stage of disease (603 subjects from 60 centers across Italy) - found a similar proportion of adenocarcinoma $(72 \%)$, but a different percentage of squamous cell carcinoma $(17 \%)$. In the selected population, molecular characterization was reported only in $64 \%$ of the cases. This low mutational detection rate is consistent with other Italian studies, such as LIFE [11], which also reported that around $60 \%$ of patients had undergone a biomarker testing. However, in this study, when considering the non-squamous population, the rate increases up to $88 \%$.

Of the Italian patients with advanced disease enrolled in the period 2011-2016 in the PIvOTAL study [17], 51\% were tested for biomarkers.

As for the prevalence of mutations found in the study population, it is consistent with AIOM data, found in "I numeri del cancro in Italia 2016" ("The numbers of cancer in Italy") [1]: $12.7 \%$ of EGFR mutations (10-15\% according to AIOM) and $4.3 \%$ of ALK translocations (4\% according to AIOM).

The therapeutic regimens used in the study population are generally aligned with international guidelines $[5,7,18,19]$. Nevertheless, 24 patients received chemotherapy as first line, despite carrying a mutation. In some of these cases, chemotherapy was administered only during the timespan needed to perform the analysis of the mutational status. In other cases, ALK mutated patients were identified, but the reimbursement of crizotinib as first line had not yet been approved (it has been active since March 2016).

Progression of disease was by far the main reason for the discontinuation of therapy, both in the first- and second-line treatment, as reported in the LIFE study [11] (on third-line treatment). Toxicity was reported to be responsible for only $6.50 \%$ and $4.12 \%$ of discontinuations of therapy in first- and second-line treatments, respectively. This is consistent with the EPICLIN study [20], in which $6 \%$ of patients discontinued first-line chemotherapy due to toxicity. On the contrary, in the LIFE study, toxicity was responsible for $14 \%$ of discontinuations of chemotherapy and $9 \%$ of discontinuations of erlotinib in third-line treatment.

With regard to the costs, this analysis concerns the impact on the expenditure incurred by the center. Drug expenditure was calculated as the price payed by the local pharmacy per single dose multiplied by the actual quantity administered. Credit notes and national management agreement are not included.

In this study, the administration of single and double chemotherapy regimens in first-line, non-mutated patients is clearly the therapeutic option which decreases the total treatment cost, whereas patients in second and third line are associated with an increase in expenditure, attributable to the introduction of immune checkpoint inhibitors (nivolumab) and to the increasing use of TKIs (crizotinib, afatinib, erlotinib, gefitinib) in mutated patients (Table VII). As a result, the cost of therapy per patient is remarkably higher above all in patients carrying ALK mutation (Table VII).

In addition, patients on treatment represent a greater expenditure (two-fold) per patient, compared to completed patients. This is likely due to an increase in the follow-up time and the introduction of new and more expensive drugs during the study period.

Since target therapy and immune checkpoint inhibitors are the main responsible for the increase in the costs of therapy (Figure 3), it was deemed necessary to perform multivariate analyses on TTNT1, TTNT2, and total costs, in order to highlight the effects of the main variables of interest, while correcting such effects for possible confounding variables (gender, age, PS, duration of therapy, smoking habit, staging, histotype, and patient's status).

Multivariate regression was performed according to LASSO methods, i.e. model predictors were selected minimizing the sum of square of residuals plus a penalty equal to the ab- 
solute value of the magnitude of coefficients. This particular type of regression is well-suited for models showing high levels of multicollinearity (e.g. patients' status and class treatment). Furthermore the algorithm automatically forces some coefficients to become zero, which is the ideal for producing simpler models. According to this methods we were able, starting with the complete model containing all possible covariates, to define the best reduced model that predict TTNT1, TTNT2, and total cost.

Multivariate analyses show that receiving a TKI after progression increases the costs in patients 1L, but also improves TTNT (Tables IX and X). These data confirm the current management of mutated patients (EGFR, ALK, and ROS1) and justify the increased costs for their treatment. In light of these data, an increase in the rate of molecular characterization is highly desirable.

Adeno patients who are eligible for maintenance after progression show a significant cost increase, due to the higher cost of pemetrexed and bevacizumab compared to the cisplatin chemotherapy and taxol (docetaxel).

As confirmed by our multivariate analysis (Table IX), the performance status affects directly the TTNT2. In fact, performance status is one of the earliest recognized prognostic factors, and is probably the strongest one [21,22].

The multivariate analysis of total costs (Table $\mathrm{X}$ ) shows that every additional month of therapy is associated with a cost increase ranging from $€ 661$ in first line up to $€ 1,383$ for patients $3 \mathrm{~L}$. This increase is due to the greater costs for the treatments used in second and third line.

The introduction of immune checkpoint inhibitors in subsequent treatment lines had the most significant impact on treatment costs in selected patients, increasing expenditure by $€$ 11,300 in patients $2 \mathrm{~L}$ and $€ 25,202$ in patients $3 \mathrm{~L}$ (Table $\mathrm{X}$ ), even though a statistically significant clinical benefit was not detected (Table IX).

For patients $3 \mathrm{~L}$, third-line treatment, due to the heterogeneity of treatment pathways followed, did not result a predictor of costs.

Among the strengths of this study, there are sample size analyzed and the real-world design, that allowed to take reliable, clear and up-to-date picture about drug utilization of the modern therapies nowadays used in the treatment of advanced NSCLC, with clear presentation of the relevant costs of this treatment.

On the contrary, the first weakness is the absence of data about progression free survival, that forced us to use TTNT1 and TTNT2 as proxies, even though the comparison of time to next treatment across treatment regimens may be affected by the bias due to the fact that many chemotherapies have fixed duration while newer drugs are administered continuously until disease progression or unacceptable toxicity. This is due also to the fact that analyses were planned after data collection. In addition, the costs associated with possible drug-related hospitalizations were not taken into account.

Due to the rapid change of drug armamentarium in the setting of lung cancer, the period of observation was relatively short, in order to focus on some specific drugs used in a not-toowide time span. For the same reason, it was not possible to consider the impact of pembrolizumab and osimertinib in the first-line treatment.

Finally, a possible bias may come from the patient population, that was probably healthier than expected, as $86 \%$ of patients had ECOG PS 0 or 1.

In 2016, the "Rapporto OsMed" ("OsMed Report") showed that antineoplastic and immunomodulatory drugs were the most expensive drugs among those supplied by the Healthcare Service in Italy in 2017 , accounting for more than $€ 5,063$ million ( $€ 83.57$ pro capite) [23].

In Italy, the mean cost of an oncologic therapy in the period 2010-2014 was over ten-fold higher than in the period 1995-1999 [24].

The aging population, together with the increase in the costs of the new therapeutic options, results in an increase in the costs borne by the health systems. Our study shows that an increased mutational selection and the introduction of immune checkpoint inhibitors in the treatment of NSCLC are likely to produce significant cost increases, while progressionfree survival gains had still to be demonstrated. Among the cost-containing measures are the implementation of prevention strategies, personalized therapies, and regulatory efforts to ensure the therapeutic appropriateness. In addition, at international level, some initiatives have been undertaken in order to share information, the identification of the best practice, the most appropriate therapeutic options, and price negotiations [25].

In 2014, the first immune checkpoint inhibitors entered the market - targeting the receptor interaction PD-1/PD-L1 - and rapidly spread in the therapeutic landscape, as confirmed by our analysis: immune checkpoint inhibitors were used more than chemotherapy as second- 
line therapy, especially in squamous cell carcinoma, since nivolumab was initially (2016) approved in squamous cell carcinoma (Table III).

Personalized therapies and new agents are more expensive than general drugs and chemotherapy, hence the raise of the sustainability issue. A proper and prompt access to these innovative drugs is mandatory, yet financial sustainability must be ensured. To this end, the Italian Regulatory Agency [26] has recently defined the criteria to evaluate whether a drug is innovative and can be reimbursed by a special cancer drug fund. These criteria are:

1. Therapeutic need;

2. Added therapeutic value;

3. Quality of evidence, or robustness of clinical trials.

Each dimension is graded, and innovativeness is recognized if the first two are categorized as "maximum" or "important" and the third as "high". Innovativeness is not recognized if the first two dimensions are considered "poor" or "absent" or if the third is "low" or "very low" (even if a "low" quality of evidence could be accepted in case of drugs for rare diseases). Intermediate situations are judged on a case-by-case basis.

In Italy, two separate funds for innovative drugs were established, one of which dedicated to oncology [27].

In 2017, the Italian Fund for innovative oncological drugs covered pertuzumab, paclitaxel, idelalisib, ibrutinib, nivolumab, and pembrolizumab [28]. Among these, nivolumab and pembrolizumab in the treatment of NSCLC are responsible for the greatest fund consumption, due to the high number of cases (followed by HER2 ${ }^{+}$breast cancer).

While the national regulatory agency defines the reimbursement rules for innovative drugs, clinicians and local decision-makers should use the highest standards of care, providing therapeutic pathways which maximize the quality of life and the clinical outcomes in all patients. As for the therapy with TKIs, our analysis confirms their real-word effectiveness and economic impact, which is mainly due to first-line options. As for the economic impact of immune checkpoint inhibitors in the treatment of NSCLC, our analysis only described the time frame in which nivolumab entered the second line and pembrolizumab was reimbursed in selected PD-L1 positive patients, both in first and second line. The evaluation of the economic impact of these innovative drugs should take into account the differential absorption of resources associated with the PD-L1 strategy.

Nivolumab was reimbursed in "all comers" second-line patients also in other EU countries, such as Switzerland [29] and the UK [30], where cost-effectiveness analyses showed that the "all comers" strategy with nivolumab was not cost-effective: the costs of nivolumab were sustainable - at local prices - only in PD-L1 positive patients. In the Italian setting, costeffectiveness analyses are lacking. However, a recently published budget impact model about the selection strategy drew similar conclusions [31]. The introduction of pembrolizumab in PD-L1 expressors received positive evaluations by studies from UK [32,33], Scotland [34], and France [35], and might have delivered a greater sustainability. Immune checkpoint inhibitors are being evaluated by FDA and EMA for new indications in a NSCLC subset of patients: their binding to a molecular biomarker characterizes all clinical trials, as well as the study of combination therapies with chemotherapy. Considering our predictors of increased treatment costs in subsequent lines (in particular, receiving immune checkpoint inhibitors in second line), the use of immune checkpoint inhibitors in selected first-line PD-L1 positive ( $>50 \%)$ patients was likely an appropriate and sustainable option for the Center. Future extension of eligibility for first-line treatment with immune checkpoint inhibitors is highly desirable in terms of improved overall survival, PFS and quality of life, and yet, the affordability of these options will require a careful evaluation.

\section{Funding}

This study was financially supported by MSD Italia Srl.

\section{Conflicts of Interests}

The authors declare they have no competing financial interests concerning the topics of this article.

\section{Acknowledgements}

We would like to thank Laura Fascio Pecetto, from SEEd Medical Publishers, and Massimiliano Povero, from AdRes Srl, who provided editorial assistance, journal styling services, and statistical analyses. Editorial services and statistical analyses were funded with an unrestricted grant by MSD Italia Srl. 


\section{REFERENCES}

1. AIOM, CCM, AIRTUM. I numeri del cancro in Italia 2016. Roma: Il Pensiero Scientifico Editore, 2016

2. Savic Prince S, Bubendorf L. Predictive potential and need for standardization of PD-L1 immunohistochemistry. Virchows Arch 2018 Sep 1 [Epub ahead of print]; https://doi. org/10.1007/s00428-018-2445-7

3. Hanna N, Johnson D, Temin S, et al. Systemic Therapy for Stage IV Non-Small-Cell Lung Cancer: American Society of Clinical Oncology Clinical Practice Guideline Update Summary. J Oncol Pract 2017; 13: 832-7; https://doi.org/10.1200/JOP.2017.026716; https:// doi.org/10.1200/JCO.2017.74.6065

4. Novello S, Barlesi F, Califano R, et al; ESMO Guidelines Committee. Metastatic nonsmall-cell lung cancer: ESMO Clinical Practice Guidelines for diagnosis, treatment and follow-up. Ann Oncol 2016; 27(suppl 5): v1-v27; https://doi.org/10.1093/annonc/mdw326

5. ESMO Guidelines Committee. eUpdate 28 June 2017: Treatment Recommendations and Revised ESMO Magnitude of Clinical Benefit Scale (ESMO-MCBS) Grading for Metastatic Non-small-cell Lung Cancer. Available at http://www.esmo.org/Guidelines/Lung-and-ChestTumours/Metastatic-Non-Small-Cell-Lung-Cancer/eUpdate-Treatment-Recommendation (last accessed November 2018)

6. ESMO Guidelines Committee. eUpdate 28 June 2017: New eUpdate featuring Updated Treatment Algorithms for Metastatic Non-small-cell Lung Cancer. Available at http://www. esmo.org/Guidelines/Lung-and-Chest-Tumours/Metastatic-Non-Small-Cell-Lung-Cancer/ eUpdate-Treatment-Algorithms (last accessed November 2018)

7. National Comprehensive Cancer Reports ${ }^{\circledR}$. NCCN Clinical Practice Guidelines in Oncology (NCCN Guidelines $\left.{ }^{\circledR}\right)$. Version 1.2018. Non-Small Cell Lung Cancer. Available at https://www.nccn.org/professionals/physician_gls/recently_updated.aspx (last accessed November 2018)

8. Crinò L, Metro G, Baldini E, et al. AIOM. Linee guida neoplasia del polmone. Edizione 2016. Available at http://www.aiom.it/C_Common/Download.asp?file=/\$Site $\$ /$ files $/ \mathrm{doc} /$ LG/2016_LG_AIOM_Polmone.pdf (last accessed November 2018)

9. Ascierto PA, Cartenì G, Gridelli C, et al. What have we learned from immunotherapy? Report from the 3rd and 4th meetings of the Campania Society of Oncology Immunotherapy (SCITO). J Immunother Cancer 2016; 4: 41; https://doi.org/10.1186/s40425-016-0144-y

10. Keytruda. Riassunto delle caratteristiche del prodotto

11. de Marinis F, Ardizzoni A, Fontanini G, et al; LIFE Study Team. Management of Italian patients with advanced non-small-cell lung cancer after second-line treatment: results of the longitudinal phase of the LIFE observational study. Clin Lung Cancer 2014; 15: 338-45. e1; https://doi.org/10.1016/j.cllc.2014.04.004

12. Agenzia Italiana del Farmaco. Determina 16 febbraio 2017. Regime di rimborsabilità e prezzo a seguito di nuove indicazioni terapeutiche del medicinale per uso umano «Opdivo». (Determina n. 252/2017). (17A01392) (GU Serie Generale n.43 del 21-02-2017). http:// www.gazzettaufficiale.it/eli/id/2017/02/21/17A01392/sg;jsessionid=SQ5FyToybg0qsmQ stokMJA_.ntc-as5-guri2a

13. Edge SB, Byrd DR, Compton CC, et al. (editors). AJCC cancer staging manual (7th ed). New York, NY: Springer; 2010

14. Agenzia Italiana del Farmaco. Determinazione 20 marzo 2008. Gazzetta Ufficiale della Repubblica Italiana - Serie Generale n. 76, 31-3-2008

15. Friedman J, Hastie T, Tibshirani R. Regularization Paths for Generalized Linear Models via Coordinate Descent. Journal of Statistical Software 2010; 33: 1-22; https://doi.org/10.18637/ jss.v033.101

16. R Core Team (2013) R: A language and environment for statistical computing R Foundation for Statistical Computing, Vienna, Austria URL http://www.r-project.org/ 
17. de Castro J, Tagliaferri P, de Lima VCC, et al. Systemic therapy treatment patterns in patients with advanced non-small cell lung cancer (NSCLC): PIvOTAL study. Eur J Cancer Care (Engl) 2017; 26; 1-15; https://doi.org/10.1111/ecc.12734

18. Masters GA, Temin S, Azzoli CG, et al. Systemic therapy for stage IV Non-Small-Cell Lung Cancer: American Society of Clinical Oncology Clinical Practice Guideline Update. J Clin Oncol 2015; 33: 3488-515; https://doi.org/10.1200/JCO.2015.62.1342

19. Narod A. Guideline on Stage IV Non-Small-Cell Lung Cancer Therapy Updated. August 14,2017

20. Carrato A, Vergnenègre $\mathrm{A}$, Thomas $\mathrm{M}$, et al. Clinical management patterns and treatment outcomes in patients with non-small cell lung cancer (NSCLC) across Europe: EPICLINLung study. Curr Med Res Opin 2014; 30: 447-61; https://doi.org/10.1185/03007995.20 13.860372

21. Jeremić B, Miličić B, Milisavljevic S. Clinical prognostic factors in patients with locally advanced (stage III) nonsmall cell lung cancer treated with hyperfractionated radiation therapy with and without concurrent chemotherapy: single-Institution Experience in 600 Patients. Cancer 2011; 117: 2995-3003; https://doi.org/10.1002/cncr.25910

22. Schild SE, Tan AD, Wampfler JA, et al. A new scoring system for predicting survival in patients with non-small cell lung cancer. Cancer Med 2015; 4: 1334-43; https://doi. org/10.1002/cam4.479

23. Osservatorio Nazionale sull'impiego dei Medicinali. L'uso dei farmaci in Italia. Rapporto Nazionale 2017. Roma: Agenzia Italiana del Farmaco, 2018. Available at http://www.aifa. gov.it/content/luso-dei-farmaci-italia-rapporto-osmed-2017 (last accessed July 2018)

24. Collicelli C, Maietta F, Castro C, et al. L'impatto dei farmaci innovativi sulla sanità italiana. $8^{\circ}$ Rapporto sulla condizione assistenziale dei malati oncologici. Roma 12-15 maggio 2016. XI Giornata nazionale del malato oncologico. Available at https:/www.favo.it/ ottavo-rapporto.html (last accessed November 2018)

25. Melazzini M. Recepire l'innovazione e garantire sostenibilità al sistema il contributo dell'AIFA. Italian Health Policy Brief 2017; 7: 1-3

26. Agenzia Italiana del Farmaco. Criteri per la classificazione dei farmaci innovativi e dei farmaci oncologici innovativi (05/04/2017). Comunicazione AIFA. Available at http:// www.agenziafarmaco.gov.it/content/criteri-la-classificazione-dei-farmaci-innovativi-e-deifarmaci-oncologici-innovativi-050420 (last accessed November 2018)

27. Curto A, Scroccaro G. Il ruolo delle regioni. Quaderni della SIF 2017; 13: 18-20

28. AIFA, 2017c. ALLEGATO 2 Fondo farmaci oncologici innovative. Available at http:// www.aifa.gov.it/sites/default/files/ALLEGATO-2_Fondo_farmaci_oncologici_innovativi_26.06.2017.pdf (last accessed December 2018)

29. Matter-Walstra K, Schwenkglenks M, Aebi S, et al.; Swiss Group for Clinical Cancer Research. A Cost-Effectiveness Analysis of Nivolumab versus Docetaxel for Advanced Nonsquamous NSCLC Including PD-L1 Testing. J Thorac Oncol 2016; 11: 1846-55; https:// doi.org/10.1016/j.jtho.2016.05.032

30. NICE appraisal, Nivolumab for previously treated locally advanced or metastatic nonsquamous non-small-cell lung cancer [ID900] https://www.nice.org.uk/guidance/indevelopment/gid-tag524/documents 1 November, 2017

31. Restelli U, Artale S, Pacelli V, et al. Budget impact analysis of a patients' selection strategy based on the expression of Programmed-Death Ligand 1 for the administration of immuno-oncologic treatments to patients affected with Non-Small Cell Lung Cancer in Italy. Clinicoeconomics Italian articles on outcomes research 2017; 12: 155-64

32. NICE 2017b. Pembrolizumab for untreated PD-L1-positive metastatic non-small-cell lung cancer. 28 June 2017. Available at https://www.nice.org.uk/guidance/indevelopment/gidta10092/documents (last accessed December 2018) 
33. NICE 2017c. Pembrolizumab for treating PDL1-positive non-small-cell lung cancer after chemotherapy. Technology appraisal guidance. Published: 11 January 2017. Available at https://www.nice.org.uk/guidance/ta428/resources/pembrolizumab-for-treating-pdl1positive-nonsmallcell-lung-cancer-after-chemotherapy-pdf-82604670410437 (last accessed December 2018)

34. Scottish Medicines Consortium. pembrolizumab 50mg powder for concentrate for solution for infusion (Keytruda $\left.{ }^{\circledR}\right)$ SMC No. (1204/17). 16 January 2017. Available at https://www. scottishmedicines.org.uk/files/advice/pembrolizumab_Keytruda_FINAL_Dec_2016_amended_020117_for_website.pdf (last accessed December 2018)

35. Haute Autorité de Santé (HAS). Commission De La Transparence Avis 3 mai 2017. Available at https://www.has-sante.fr/portail/upload/docs/evamed/CT-15634_KEYTRUDA_PIC_EI_ poumon_Avis3_modifiele03052017_CT15634.pdf (last accessed December 2018) 